\title{
Editorial
}

\section{Given the complexity of the human genome, can 'personalised medicine' or 'individualised drug therapy' ever be achieved?}

Ever since the Human Genome Project began in October 1990, hundreds of extremely optimistic reviews have been written, suggesting that 'personalised medicine was just around the corner' or that 'individualised drug therapy was only a few months away'. Some medical researchers even began to launch DNA-typing companies, expecting large profits very soon.

Contrary to this point of view and opposing such hype, several of us have steadfastly insisted that the genome is far too complex for us to understand at the present time. ${ }^{1-5}$ In fact, the 2003 Nebert et al. review ${ }^{1}$ was initially rejected by a very highcitation-indexed journal; the author suspects that one or more of the reviewers may have had financial interests in DNA-assay companies.

Can some number of DNA variant sites (genotype) really be associated - 100 per cent of the time - with the diagnosis of a complex disease such as obesity, or schizophrenia, or coronary artery disease or type-2 diabetes (phenotype)? Can some number of single nucleotide polymorphisms (SNPs) (genotype) really be associated - 100 per cent of the time - with the prediction of a drug response (phenotype)? Ideally, a 100 per cent level of success in genotype-phenotype association studies is what physicians require for personalised medicine or individualised drug therapy to be successful.

Sequencing of the entire human genome, the HapMap Consortium (Phases I, II and III,
2003-2009) has now identified more than 11 million SNPs in 1,115 individuals from 11 populations worldwide having minor allele frequencies (MAFs) of $\geq 5$ per cent. Increasingly, cost-effective high-throughput genotyping technologies leading to genome-wide association (GWA) studies have demonstrated the requirements needed to separate true associations from the plethora of publications dealing with false positives. 'Third-generation DNA sequencing' promises, in 2009 and 2010, to be able to sequence dozens of human genomes per month.

The Encyclopedia of DNA Elements (ENCODE) Pilot Project, covering $\sim 1$ per cent of the human genome (2004-2007), however, has shown us that we are no longer even certain what a 'gene' is. ENCODE discovered multiple transcription start sites, complexities of histone modifications and chromatin remodeling 'beyond our wildest expectations', regulatory elements in trans on one chromosome controlling transcripts on a different chromosome, and an incredible $>60$ per cent of all DNA (both gene-rich and gene-desert regions) highly conserved between primates and pufferfish. There are dozens of reasons why an unequivocal genotype (also an unequivocal phenotype) is virtually impossible to achieve in current limitedsize studies of human populations. ${ }^{1-5}$ This problem (of insufficiently stringent criteria) has led to decreased statistical power and, consequently, 
equivocal interpretation of most genotype-phenotype association studies. Comparing 'monogenic', high-penetrance predominantly monogenic (hPpM) pharmacogenetic disorders, and 'complex' diseases, the percentage that any one gene might contribute to the phenotype varies: $>90$ per cent, 15-25 per cent, and $<1$ per cent, respectively. ${ }^{2}$

The human genome is still largely a mystery. RNA interference (RNAi) genes number in the thousands. Many large intervening non-coding RNAs (lincRNAs), ranging in size from 2.3 to 17.2 kilobases, exist in gene-desert regions, highly conserved and with no known function. We know that genetic systems evolve over millions of years to a point of maximum stability in the face of genetic and environmental perturbation. Yet, waves of genetic change accompanying the origin of the Homo genus, and subsequent migration around the globe, have disrupted this stable equilibrium with an explosion (from hunter-gatherer to farmer to urban living) in the past 6,000 years. Within the past $\sim 300$ years, dramatic cultural changes (striking dietary shifts, tobacco smoking, air pollution, altered pathogen exposure, taking drugs [prescribed, over-the-counter, recreational] and psychological stress) have undoubtedly effected epigenetic changes in our genome.

In the 16th April 2009 edition of the New York Times, suddenly and unexpectedly there appeared an article titled 'Study of genes and diseases at an impasse'. The first sentence of the article stated: 'The era of personal genomic medicine may have to wait'. This newspaper article referred to a review that was about to appear in the 23rd April 2009 issue of the New England Journal of Medicine, ${ }^{6}$ followed by three 'Perspectives'. ${ }^{7-9}$ Each article, in its own way, described the complexities being encountered in the human genome and offered possible solutions, but agreed that personalised medicine or individualised drug therapy was not yet close at hand. None of the previous reviews by Nebert and colleagues was cited.

Where are we now? For individualised drug therapy, it is predicted that, while some adverse drug reactions/efficacy traits — associated directly with plasma drug and/or urinary metabolite levels might follow hPpM (15-25 per cent) inheritance of individual genes, the vast majority will be no different from 'complex diseases' $(<1$ per cent) inheritance of hundreds or thousands of genes. We must appreciate that virtually all drug responses just like all complex diseases - represent gradients, reflecting quantitative trait loci (QTLs); accordingly, these responses can be evaluated by alterations in genetic network expression (eQTLs), metabolism (mQTLs) and perhaps protein (pQTLs) profiles. It is predicted that the future of individualised drug therapy and personalised medicine will involve a combination of these, rather than DNA tests alone.

Daniel W. Nebert
Genome Update Editor
University of Cincinnati Medical Center
Cincinnati
OH 45267-0056
USA

\section{References}

1. Nebert, D.W., Jorge-Nebert, L.F. and Vesell, E.S. (2003), 'Pharmacogenomics and "individualized drug therapy": High expectations and disappointing achievements', Am. J. Pharmacogenomics Vol. 3, pp. $361-370$.

2. Nebert, D.W. and Vesell, E.S. (2004), 'Advances in pharmacogenomics and individualized drug therapy: Exciting challenges that lie ahead', Eur. J. Pharmacol. Vol. 500, pp. 267-280.

3. Nebert, D.W. (2005), 'Interindividual susceptibility to environmental toxicants - A current assessment', Toxicol. Appl. Pharmacol. Vol. 207, pp. 34-42.

4. Nebert, D.W. and Vesell, E.S. (2006), 'Can personalized drug therapy be achieved? A closer look at pharmaco-metabonomics', Trends Pharmacol. Sci. Vol. 27, pp. 580-586.

5. Nebert, D.W., Zhang, G. and Vesell, E.S. (2008), 'From human genetics and genomics to pharmacogenetics and pharmacogenomics: Past lessons, future directions', Drug Metab. Rev. Vol. 40, pp. 1-38.

6. Hardy, J. and Singleton, A. (2009), 'Genome-wide association studies and human disease', N. Engl. J. Med. Vol. 360, pp. 1759-1768.

7. Goldstein, D.B. (2009), 'Common genetic variation and human traits', N. Engl. J. Med. Vol. 360, pp. 1696-1698.

8. Hirschhorn, J.N. (2009), 'Genome-wide association studies Illuminating biologic pathways', N. Engl. J. Med. Vol. 360, pp. 1699-1701.

9. Kraft, P. and Hunter, D.J. (2009), 'Genetic risk prediction - Are we there yet?', N. Engl. J. Med. Vol. 360, pp. 1701-1703. 